\title{
Os sistemas de informação na prática do médico de família: onde está a interoperabilidade?
}

Ana Paula Pinheiro*

\section{RESUMO}

Os sistemas de informação clínica são, neste momento, essenciais nas unidades de saúde, permitindo a partilha de informação, com melhoria da eficiência e qualidade dos cuidados de saúde. Este trabalho pretende analisar os diversos sistemas de informação com que um médico de família, no Serviço Nacional de Saúde Português, pode lidar diariamente na sua prática clínica, bem como a sua interoperabilidade. A autora utiliza a sua própria experiência como médica de família numa Unidade de Saúde Familiar para descrever esta realidade. A interoperabilidade constatada entre os vários sistemas é mínima, o que leva ao desgaste dos profissionais e ao desperdício de recursos.

Palavras-chave: Sistemas de informação; Cuidados de saúde primários; Interoperabilidade

\section{INTRODUÇÃO}

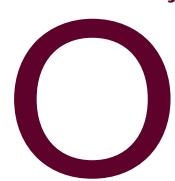

s registos clínicos em papel têm limitações inerentes à sistematização da informação clínica, não permitindo um acesso rápido ao número crescente de dados. São necessárias ferramentas que transformem os processos de trabalho e que suportem a inovação na prestação de cuidados de saúde. ${ }^{1}$ Os registos clínicos informáticos permitiram superar estas limitações, com flexibilidade dos formatos e das entradas. ${ }^{2-4}$

Atualmente, em Portugal, na maioria das instituições, os diversos profissionais clínicos de uma mesma instituição conseguem aceder aos dados clínicos nos diversos contactos do utente com a mesma.

Os avanços tecnológicos permitiram o desenvolvimento progressivo dos sistemas de informação. No entanto, os problemas persistem e têm vindo a ser identificados. Um dos principais problemas da informática médica é a capacidade de partilhar a informação contida nos processos clínicos. Existem vários sistemas dispersos e heterogéneos que dificultam o acesso à infor-

*Coordenadora da USF Viseu-Cidade

Diretora do Núcleo de Internato de Medicina Geral e Familiar de Viseu

Membro da Comissão para a Formação Pré e Pós-graduada do Centro Académico Clínico das Beiras (CACB)

Assessora para acompanhamento do Protocolo de Ensino Médico com a Universidade da Beira Interior mação clínica por profissionais de diferentes instituições..$^{5-6}$

O médico de família debate-se com diversos desafios resultantes das lacunas existentes nos sistemas de informação. A autora identifica estes problemas, com base na literatura disponível e na sua própria experiência. Apresenta ainda algumas propostas de melhoria neste âmbito.

\section{INTEROPERABILIDADE}

Em saúde, a interoperabilidade é a capacidade de diferentes sistemas de informação comunicarem, trocarem e usarem a informação que foi partilhada. Os esquemas de partilha de dados deveriam permitir o intercâmbio dos mesmos entre clínicos, laboratórios, hospital e farmácia. ${ }^{3,7} \mathrm{~A}$ ausência de interoperabilidade entre as organizações de saúde conduz ao uso ineficaz dos recursos existentes em cada organização. ${ }^{8-9}$ Sendo assim, o caminho correto a seguir será o da interoperabilidade, de modo a permitir que todos os profissionais de saúde envolvidos tenham acesso a toda a informação disponível, independentemente do local. A nível nacional têm havido vários esforços no sentido da interoperabilidade. Internacionalmente têm sido implementadas redes regionais e nacionais com a integração de dados de saúde em número crescente. ${ }^{10}$ 
Assistimos ainda a uma transição no sentido da agregação de unidades de saúde, facilitando a sua ligação em rede, levando a inevitáveis investimentos na integração entre sistemas existentes. Para o possibilitar várias soluções tecnológicas distintas coexistem, permitindo a integração de um crescente volume de dados, fazendo uso de diferentes normas e arquiteturas. ${ }^{11}$

Os projetos de integração regional são fundamentalmente entre hospitais e cuidados primários. ${ }^{11} \mathrm{Um}$ resumo da informação do doente tem sido encarado como a forma mais adequada ao estabelecimento de interoperabilidade entre distintos sistemas. Este resumo deverá incluir a história clínica, alergias, problemas ativos, resultados de exames complementares e medicação habitual, sendo necessária investigação adicional em relação à quantidade de informação estruturada que é necessária. ${ }^{12-13}$

Na Europa, a interoperabilidade também tem sido abordada pelos políticos. A União Europeia lançou em 2004 o European eHealth Action Plan ${ }^{14} \mathrm{e}$ a Comissão Europeia tem financiado projetos na área da interoperabilidade semântica (SemanticHealth e RIDE-Roadmapfor Interoperability of eHealth Systems). O SemanticHealth é uma ferramenta específica de suporte para desenvolver a nível europeu e mundial um roteiro para a implementação e investigação na área da saúde e das TIC, com foco nas questões da interoperabilidade semântica.

Em Portugal, a maior parte dos serviços de saúde é suportado por um conjunto de ilhas de sistemas fragmentados de informação que foram sendo adquiridos sem qualquer estratégia.

Na década de 1980, os sistemas de informação em saúde eram sistemas informáticos isolados, enquanto na década de 1990 passaram a ser mais integrados. Os cuidados de saúde primários e os secundários tiveram a primeira ligação suportada pelo SINUS-SONHO no ano $2000 .{ }^{15}$ Desde abril de 2006 os centros de saúde começaram a ser interligados aos hospitais através do ALERT ${ }^{\circledR}-P 1$. Esta iniciativa, inserida no programa SIMPLEX, «consulta a tempo e horas», permite a marcação e priorização das consultas de especialidade nos hospitais. Posteriormente surgiram o SClínico e o PDS, que permitiram uma maior interligação entre os cuidados primários e secundários.

Um bom sistema de saúde faz um aproveitamento eficiente dos recursos existentes e presta cuidados de saúde de qualidade. ${ }^{16} \mathrm{O}$ sistema de saúde deve funcionar como um todo, necessitando de ferramentas que transformem os processos de trabalho e que suportem a inovação na prestação de cuidados de saúde. ${ }^{17}$ Todas as atividades em saúde estão relacionadas com a busca e o uso da informação. Logo, quanto melhor os sistemas informatizados funcionarem quanto ao registo, armazenamento e disponibilização da informação melhor será a informação e a qualidade na tomada de decisão. ${ }^{18}$

Os sistemas de informação em saúde são essenciais para a modernização dos serviços, com benefícios evidentes no armazenamento, organização e acesso à informação. Há um ganho na qualidade do trabalho. ${ }^{19-20}$

\section{SISTEMAS DE INFORMAÇÃO NA PRÁTICA DE UM MÉDICO DE FAMÍLIA}

A prática diária de um médico de família é um tumulto de cliques em diversas plataformas informáticas, a maioria delas sem interligação entre si. No total é necessário saber lidar com pelo menos nove aplicações informáticas, sendo necessário trabalhar com cada uma delas de forma intuitiva (SClínico, PEM, ALERT, PDS, SiiMA rastreios, TAOnet, SAPA, SICO, SCGTD, MIM@UF, SINAVE, entre outras). Irei debruçar-me apenas sobre as mais relevantes para a prática clínica.

O SClínico é a base da consulta nos cuidados de saúde primários, um sistema de informação clínica desenvolvido pelos SPMS (Serviços Partilhados do Ministério de Saúde) a partir de duas aplicações anteriores: o SAM (Sistema de Apoio ao Médico) e o SAPE (Sistema de Apoio à Prática de Enfermagem). Tem tido várias atualizações sucessivas, sendo uma aplicação única comum a todos os prestadores de cuidados de saúde primários, nomeadamente médicos, enfermeiros, fisioterapeutas, psicólogos, entre outros. O SClínico surgiu na estratégia definida pelo Ministério da Saúde para a área de informatização clínica do Sistema Nacional de Saúde (SNS), com o intuito de maior uniformização dos registos clínicos. A homogeneização das práticas a nível nacional aumenta a eficácia e eficiência de atuação dos profissionais de saúde. Permite também a partilha de informação pela equipa multidisciplinar que acompanha o utente, com poupança de recursos e melhoria da qualidade de atendimento.

Para aceder a esta plataforma é necessário colocar o número mecanográfico e uma password. 
Infelizmente, apesar de ser a ferramenta diária de uso na maioria das unidades de saúde de cuidados primários, ainda não o é a nível hospitalar em todo o país. Muitos dos registos das consultas hospitalares em alguns locais são efetuados no ALERT, não havendo interface acessível para os cuidados primários. Origina perda de informação relevante para o médico de família que, na maioria das situações, foi o responsável pelo encaminhamento para outra especialidade para esclarecimento. Fica a aguardar eventual retorno de informação clínica, que poderá nunca ocorrer. Seria importante as entidades responsáveis tomarem consciência das limitações que daqui surgem, com potencial perda de ganhos em saúde para o utente, o elemento primordial em todo este processo.

Um outro passo importante seria a desmaterialização de todos os Meios Complementares de Diagnóstico (MCDT), além da desmaterialização do retorno da informação clínica com receção dos resultados editáveis, com anuência do utente. Pouparia muito tempo da consulta, essencial para o contacto com o utente.

A PEM (Prescrição Eletrónica Médica) é outra plataforma diariamente usada pelo médico de família. Trata-se de uma ferramenta para a prescrição médica, com ligação direta às farmácias e possibilidade de emissão de receituário por via eletrónica. Com as atualizações recentes o utente saiu beneficiado, existindo diversos sistemas para emissão de uma receita: em papel, para o telemóvel e/ou para o endereço eletrónico do utente.Também permite a prescrição de cuidados respiratórios domiciliários, para já apenas em papel. Apesar de a receita por via eletrónica ser um avanço, todo o processo poderia ser mais simplificado. Por cada receita prescrita é necessário colocar uma senha e fazer três cliques. Este é um processo moroso, com perda de tempo útil de consulta.

É evidente que traz várias vantagens, nomeadamente para os utentes, como a possibilidade de poderem optar por aviar todos os produtos prescritos ou apenas parte deles, sendo possível levantar os restantes em diferentes estabelecimentos e em datas distintas e receberem por SMS os códigos de acesso, dispensa, direito de opção e o número da prescrição. Permite ainda conhecer toda a medicação prescrita a determinado doente, facilitando a decisão médica. A receita eletrónica também permite um melhor planeamento e gestão das necessidades e custos dos medicamentos, bem como reduzir as oportunidades de fraude pela invalidação automática das receitas aviadas.

Apesar dos inúmeros benefícios que a sua implementação já trouxe até à data, ainda há muito a desenvolver. Seria interessante que este sistema tivesse a capacidade de integração e cruzamento de alguma informação clínica, como as reações alérgicas/intolerâncias registadas no processo clínico, com alertas no ato da prescrição. Também deveria alertar para as interações medicamentosas ou erros na dosagem, assim como a necessidade de ajuste de dose/ausência de prescrição em casos de insuficiência hepática ou renal, reduzindo a probabilidade de erros.

O ALERT ${ }^{\circledR}$ P1 é utilizado para a referenciação hospitalar de um utente a partir dos cuidados de saúde primários. O médico de família pode aceder a este sistema através do SClínico, o que se torna mais rápido e prático ao longo de uma consulta, ou diretamente na plataforma do ALERT após introdução de username e password (mais complexo e moroso). Este tipo de sistema permite a simplificação de procedimentos, juntando a informação clínica ao pedido de consulta, estabelecendo prioridades com base em critérios clínicos e informando o utente sobre o tempo de espera.

Tem a vantagem de permitir reencaminhar, de forma automática, um pedido para uma nova especialidade ou hospital até ao momento da avaliação do pedido a nível hospitalar, sem ter que refazer toda a informação de novo. Caso haja devolução do pedido hospitalar com indicação de reencaminhamento para outra especialidade também é possível copiar a informação clínica enviada previamente acedendo ao $\mathrm{Pl}$ anterior através do SClínico, o que facilita todo o processo.

Uma das deficiências mais graves da referenciação pelo ALERT é a falta de resposta de retorno. Seria útil que, após a realização da consulta hospitalar pedida, a informação de retorno também fosse inserida na mesma plataforma de referenciação com um sistema de alerta. Assim, toda a informação estaria disponível em ambos os sentidos.

É também fundamental que este sistema seja utilizado obrigatoriamente para o acesso de qualquer pessoa às consultas hospitalares: pedidos dos cuidados de saúde primários (CSP), pedidos locais (familiares e amigos de profissionais do hospital), referenciações internas entre serviços do mesmo hospital ou referencia- 
ções entre hospitais diferentes. Deve ser assegurada esta possibilidade em qualquer local do circuito de referenciação (consulta externa ou serviço de urgência). Apenas desta forma são aplicados os mesmos critérios de prioridade, sem iniquidades entre utentes.

A PDS (Plataforma de Dados da Saúde) permite que todos os profissionais de saúde (médicos e enfermeiros) dos cuidados primários e hospitais do SNS tenham acesso à informação clínica do utente. $O$ acesso ao PDS foi uma mais-valia na prática diária do médico de família, possibilitando aceder aos resultados dos exames complementares de diagnóstico realizados no hospital de referência. Evita assim uma duplicação de exames, com redução dos custos para o SNS e maior comodidade do utente. Também permite aceder aos relatórios de alta dos internamentos de forma simultânea. É o início da interoperabilidade entre serviços de saúde regionais, que almejamos ver alargada de forma mais plena e a nível nacional. Segundo o relatório OCDE 2015 sobre a QuALIDADE DOS CUIDADOS DE SAÚdE EM PORTUGAL, Portugal poderá ser um exemplo a seguir se prosseguirem os desenvolvimentos nesta área.

O SiiMA rastreios é um sistema de informação para gestão de Programas de Rastreio Populacionais. Inclui todo o circuito do rastreio, desde o convite para o exame até ao tratamento e seguimento. Inclui os módulos de Rastreio do Cancro do Colo do Útero, Rastreio do Cancro Colorretal, Rastreio do Cancro da Mama e Rastreio da Retinopatia Diabética. Na região centro, ao nível dos CSP, apenas estão a ser utilizados os dois primeiros módulos. Seria de ponderar a introdução dos restantes módulos na prática diária, permitindo ausência de resultados em papel. Outra solução ainda melhor seria a migração dos resultados diretamente para o SClínico, para o processo clínico individual de cada utente, sem necessidade de introdução dos mesmos e com o benefício de redução do tempo despendido.

\section{CONCLUSÃO}

Os sistemas de informação são de extrema importância no trabalho diário do médico de família. A possibilidade de cópia e edição de dados, transmissão instantânea de informação, arquivo em espaço físico negligenciável e possibilidade de backup são algumas das vantagens inegáveis. Na realidade do médico de família estas vantagens são já bem percetíveis, sendo a cobertura da sua atividade por sistemas informáticos a suficiente para relegar o registo em papel para um plano vestigial.

Talvez a principal vantagem que se procure obter com estes sistemas seja a partilha de informação, evitando redundâncias e desperdício de recursos. É essencial que essa partilha ocorra de forma plena e eficaz. Isto deveria ser relativamente fácil de conseguir num sistema que é inteiramente público e, portanto, com uma única tutela. No entanto, da análise que realizou aos sistemas de informação em uso, a autora constatou que a interoperabilidade existente é mínima e claramente insatisfatória.

O número de programas em uso é excessivo e a interação entre as suas bases de dados é, em muitos casos, nula. A introdução de dados de forma redundante é uma constante e a consulta de dados nos sistemas de outras instituições revela-se muitas vezes impossível. $\mathrm{O}$ facto de a plataforma de registo da atividade clínica diária do médico de família não ser única a nível nacional (ou, pelo menos, ter interoperabilidade assegurada a nível nacional) revela o quanto ainda temos de caminhar no sentido da otimização dos recursos.

A PDS apresenta-se como um bom exemplo de uma plataforma que tenta aumentar os níveis de partilha em termos nacionais mas, dada a multiplicidade de programas cujos dados teria de agregar, os resultados práticos são ainda muito precários.

A discussão em relação a este tema parece extemporânea considerando o avanço que revelam múltiplas outras áreas, desde as redes sociais até à administração tributária. Atualmente dever-se-iam discutir estratégias de otimização de partilha de informação e não ainda discutir as estratégias para a sua implementação.

Os profissionais de saúde poderão contribuir alertando as entidades responsáveis para as dificuldades sentidas na sua prática, o que permitirá uma melhoria progressiva de todo o processo. A tutela pode e deve contribuir criando uniformidade no sistema e promover a interoperabilidade, tornando-a obrigatória nos programas aceites para uso pelos profissionais.

\section{REFERÊNCIAS BIBLIOGRÁFICAS}

1. Dogac A, Namli T, Okcan A, Laleci G, Kabak Y, Eichelberg M. Key issues of technical interoperability solutions in eHealth and the RIDE [Internet]. In: ProjectIn eChallenges Conference, The Hague (The Netherlands), October 2007. Available from: http://citeseerx.ist.psu.edu/view- 
doc/summary?doi=10.1.1.627.9561

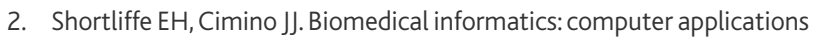
in health care and biomedicine. 4th ed. London: Springer; 2014. ISBN 9781447144748

3. Marin HF. Sistemas de informação em saúde: considerações gerais [Health information system: general considerations). J Health Inform. 2010;2(1):20-4. Portuguese

4. Sandi AA. A importância dos sistemas de informação em saúde: estudo de caso na USF CelaSaúde [Dissertation]. Coimbra: Faculdade de Economia, Universidade de Coimbra; 2015.

5. Kitsiou S, Manthou V, Vlachopoulou M. A framework for the evaluation of integration technology approaches in healthcare. In: Proceedings of the IEEE in Information and Communication Technologies in Bioinformatics, loannina (Greece), October 28-30, 2006.

6. Grimson S, Stephens G, Jung B, Grimson W, Berry D, Pardon S. Sharing health-care records over the Internet. IEEE Internet Computing. 2001; 5(3):49-58.

7. HIMSS. Health information and technology resource library [homepage]. Available from: www.himss.org/library/interoperability-standards/whatis?navltemNumber $=17763$

8. Orlova AO, Dunnagan M, Finitzo T, Higgins M, Watkins T, Tien A, et al. Electronic health record - public health (EHR-PH) system prototype for interoperability in 21st century healthcare systems. AMIA Annu Symp Proc. 2005:575-9.

9. Cavalcante RB, Pinheiro MM. Política nacional de informação e informática em saúde: avanços e limites atuais. Perspect Gestão Conhecimento. 2011;1(2):91-104.

10. Haux R. Health information systems: past, present, future. Int J Med Inform. 2006;75(3-4):268-81.

11. Cruz-Correia RJ, Vieira-Marques PM, Ferreira AM, Almeida FC, Wyatt JC, Costa-Pereira AM. Reviewing the integration of patient data: how systems are evolving in practice to meet patient needs. BMC Med Inform Decis Mak. 2007;7:14.

12. Häyrinen K, Saranto K, Nykänen P. Definition, structure, content, use and impacts of electronic health records: a review of the research literature. Int J Med Inform. 2008;77(5):291-304.

13. Guedes AS. A aceitação do registo de saúde electrónico pelos profis- sionais de saúde das instituições hospitalares [Dissertation]. Lisboa: Escola Nacional de Saúde Pública, Universidade Nova de Lisboa; 2011.

14. European Commission. eHealth action plan 2012-2020: innovative healthcare for the 21st century [homepage]. Brussels: European Commission; 2012 [cited 2016 Dec 2]. Available from: https://ec.europa.eu/ digital-single-market/en/news/ehealth-action-plan-2012-2020-innovative-healthcare-21st-century

15. Castanheira J. Jornadas Saúde 2005: processos de integração e partilha de informação. [cited 2016 Dec 2]. Available from: http://www. algebrica.pt/i_s/bo2/data/upimages/Castanheira-Jornadas.pdf

16. Rigby M, Roberts R, Williams J, Clark J, Savill A, Lervy B, et al. Integrated record keeping as an essential aspect of a primary care led health service. BMJ. 1998;317(7158):579-82.

17. Poissant $L$, Pereira J, Tamblyn R, Kawasumi $Y$. The impact of electronic health records on time efficiency of physicians and nurses: a systematic review. J Am Med Inform Assoc. 2005;12(5):505-16.

18. Stumpf M, Fisher PD, Freitas HM, Becker JL. Um modelo de integração de informações para o apoio à decisão na gestão da assistência à saúde. Porto Alegre: Programa de Pós-Graduação em Administração, Universidade Federal do Rio Grande do Sul; 1998.

19. Ribeiro LF. Interoperabilidade nos sistemas de informação de saúde: das convicções à realidade [Dissertation]. Porto: Faculdade de Medicina, Universidade do Porto; 2011.

20. Ribas MJ. Desafios e oportunidades do sistema informático [Opportunities and challenges in our informatic systems]. Rev Port Med Geral Fam. 2016;32(5):298-9. Portuguese

\section{CONFLITO DE INTERESSES}

A autora declara não ter quaisquer conflitos de interesse.

\section{ENDEREÇO PARA CORRESPONDÊNCIA}

Ana Paula Pinheiro

E-mail: ana_paulapinheiro@hotmail.com

http://orcid.org/0000-0002-8523-7714

Recebido em 16-09-2017

Aceite para publicação em 23-03-2018

\section{ABSTRACT}

\section{INFORMATION SYSTEMS IN THE PRACTICE OF THE FAMILY PHYSICIAN: WHERE IS THE INTEROPERABILITY?}

At the moment, clinical information systems are essential in Health Units, allowing the sharing of information, with improvement of efficiency and quality of health care. This work intends to analyze the various information systems witch a family physician, in the Portuguese National Health Service, can handle daily in their clinical practice, as well as their interoperability. The author uses her own experience as a family physician in a Family Health Unit to describe this reality. Interoperability established between the various systems is minimal, which leads to professional wear and waste of resources.

Keywords: Information systems; Primary healthcare; Interoperability 